\title{
Long-term evolution of asteroids in the 2:1 Mean Motion Resonance
}

\author{
Despoina K. Skoulidou, Kleomenis Tsiganis and Harry Varvoglis \\ Section of Astrophysics, Astronomy \& Mechanics, Department of Physics, \\ Aristotle University of Thessaloniki, 54124 Thessaloniki, Greece \\ email: dskoulid@physics.auth.gr, tsiganis@auth.gr, varvogli@physics.auth.gr
}

\begin{abstract}
The problem of the origin of asteroids residing in the Jovian first-order mean motion resonances is still open. Is the observed population survivors of a much larger population formed in the resonance in primordial times? Here, we study the evolution of 182 long-lived asteroids in the 2:1 Mean Motion Resonance, identified in Brož \& Vokrouhlický (2008). We numerically integrate their trajectories in two different dynamical models of the solar system: (a) accounting for the gravitational effects of the four giant planets (i.e. 4-pl) and (b) adding the terrestrial planets from Venus to Mars (i.e. 7-pl). We also include an approximate treatment of the Yarkovksy effect (as in Tsiganis et al. 2003), assuming appropriate values for the asteroid diameters.
\end{abstract}

Keywords. asteroids, methods: numerical, resonances, Yarkovsky effect

\section{Introduction}

The 2:1 Mean Motion Resonance with Jupiter is one of the famous Kirkwood gaps. In the framework of the 3-body problem, Lemaittre \& Hernard (1990) found only a small chaotic region in low-eccentricities and Morbidelli \& Moons (1993), using an improved 3-body problem, found the borders of secular resonances embedded in the 2:1 MMR. Moons et al. (1998) and Nesvorný \& Ferraz-Mello (1997) mapped the structure of the 2:1 MMR in detail, using a semi-analytical method and extensive $N$-body simulations. Long-term evolution of the observed population in the resonance, in the framework of the giant planets model, has been studied by Brož \& Vokrouhlický (2008). In this paper, we study the 'stability' of the 2:1 long-lived population in different dynamical models.

\section{Long-term evolution in the 2:1 MMR}

We performed long-term orbital integrations of the 2:1 'stable' population, for a time equivalent to 3 Gy using the SWIFT package (Levison \& Duncan 1994) and various time-steps, taking initial conditions from ASTDYS $\dagger$. We computed asteroid diameters, assuming the same albedo $\left(p_{V}=0.06\right)$ for all bodies and taking absolute magnitude values $(H)$ from Brož and Vokrouhlický (2008).

Fig. 1 shows a plot of the fraction $N(>t) / N_{i}$ of asteroids, that remain after time $t$, vs. $t$, for all the considered models. We assume a distribution law $N(>t) / N_{i}-C=e^{-b t} \ddagger$. We find that $C=C_{\max }$ is only $19 \%$ of the initial population for the 4 -pl model, $14 \%$ for the 7 -pl model, and $\approx 0 \%$ for either model that includes the Yarkovsky effect. The e-folding time, $\frac{1}{b}$, decreases from $1.70 \pm 0.02$ Gy (simple 4-pl model) to $0.89 \pm 0.01$ Gy (7-pl model with Yarkovsky).

$\dagger$ http://hamilton.dm.unipi.it/astdys/

$\ddagger$ When correlation coefficient, $r^{2}$, reaches its maximum then $C=C_{\text {max }}$ represents the percentage of the bodies that is expected to remain in the resonance as $t$ goes to infinity. 

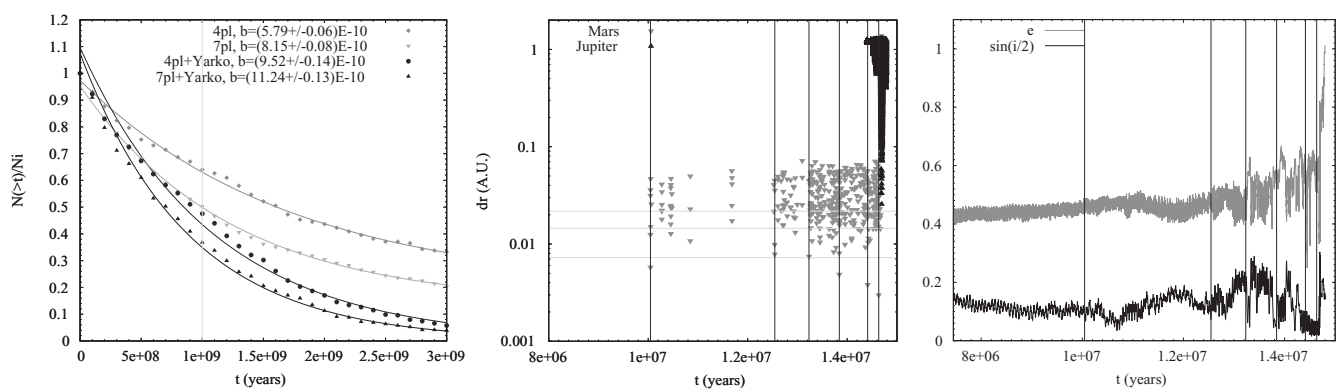

Figure 1. (left) $N(>t) / N_{i}$ vs. $t$ for all the considered models and the best-fitting curve for $C_{\max }$, (middle) close encounters with planets of asteroid 2005AO24 in the 7-pl model, (right) $e$ vs. $t$ and $\sin (i / 2)$ vs. $t$ of asteroid $2005 \mathrm{AO} 24$ at the same time interval.

\section{Close encounters and escape}

Asteroids residing in the quasi-stable core of the 2:1 resonance are, in principle, phaseprotected by encounters with Jupiter, if their orbits are not strongly modified by the secular resonances (Morbidelli \& Moons 1993). We find that many bodies (Fig. 1) have successive encounters with Mars that help in breaking up the phase-protection and extracting bodies from the resonance. The possibility of a 2:1 asteroid becoming Marscrosser was first mentioned by Wisdom (1987).

\section{Conclusions}

We found that $\sim 67 \%$ of the population manage to escape within 3 Gyr in the 4-pl model, $\sim 80 \%$ in the 7 -pl model and $\sim 95 \%$ in either model that includes the Yarkovsky effect, but the evolution is accelerated when the terrestrial planets are also considered. However, we do not find any significant contribution of the terrestrial planets to the evolution of the size distribution. We find that many asteroids have successive encounters with Mars and the other terrestrial planets, which break the phase-protection mechanism of the 2:1 MMR with Jupiter. The problem of whether the 2:1 quasi-stable population is primordial or captured in later stages of solar system evolution is still open. Clearly, a much larger initial population with a much steeper size frequency distribution is needed to explain the observed population.

\section{Acknowledgments}

The work of H. V. is co-financed by the European Union (European Social Fund - ESF) and Greek national funds through the Operational Program "Education and Lifelong Learning" of the National Strategic Reference Framework (NSRF) - Research Funding Program: Thales. Investing in knowledge society through the European Social Fund.

\section{References}

Brož M. \& Vokrouhlický D. 2008, MNRAS 390, 715

Lemaître, A. \& Hernard, J. 1990, Icarus 83, 391

Levison, H. \& Duncan, M. 1994, Icarus 108, 18

Moons, M., Morbidelli, A. \& Migliorini, F. 1998, Icarus 135, 458

Morbidelli, A. \& Moons, M. 1993, Icarus 102, 316

Nesvorný D. \& Ferraz-Mello, S. 1997, Icarus 130, 247

Tsiganis, K., Varvoglis, H., \& Morbidelli, A. 2003, Icarus 166, 131

Wisdom, J. 1987, Icarus 72, 241 